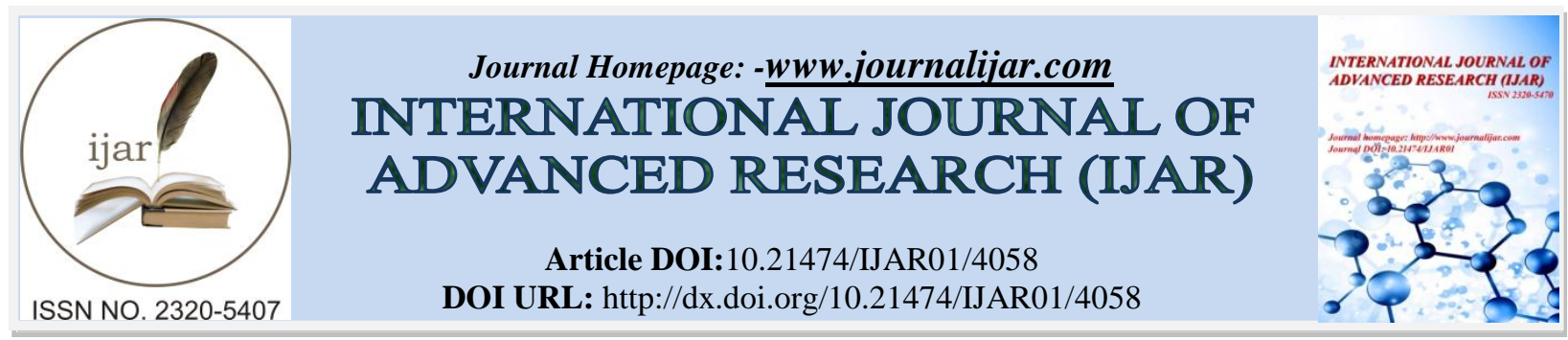

RESEARCH ARTICLE

\title{
QUANTITATIVE AND QUALITATIVE MAXILLARY JAW BONE ASSESSMENT USING CONE BEAM COMPUTED TOMOGRAPHY OF A DIABETIC VERSUS A NONDIABETIC SAMPLE OF EGYPTIAN POPULATION.
}

\author{
Sarah M. Kenawy ${ }^{1}$, Dina M. El Beshlawy² and MushiraM. Dahaba ${ }^{3}$. \\ 1. Assistant Lecturer, Oral and Maxillofacial Radiology Department, Faculty of Dentistry, Cairo University, \\ Cairo, Egypt. \\ 2. Associate Professor, Oral and Maxillofacial Radiology Department, Faculty of Dentistry, Cairo University, \\ Cairo, Egypt. \\ 3. Professor, Oral and Maxillofacial Radiology Department, Faculty of Dentistry,Cairo University, Cairo, Egypt.
}

\section{Manuscript Info}

\section{Manuscript History}

Received: 22 February 2017

Final Accepted: 26 March 2017

Published: April 2017

Key words:-

Diabetes Mellitus; Cone Beam

Computed Tomography;Bone Density; Maxilla.

\begin{abstract}
Background and purpose:Evaluation of maxillary bone is very essential for the diagnosis, treatment planning, and management of maxillofacial procedures. This study aimed to evaluate the maxillary jaw bone quality and quantity in a sample of Egyptian patients with diabetes mellitus (DM) compared to another nondiabetic sample using Cone Beam Computed Tomography (CBCT) and also to correlate between the maxillary jaw bone quality and quantity and other factors as glycated haemoglobin (HbA1c) values, disease duration, type of medication, extraction period, and gender.Methods:After approving the study protocol by the local Ethics Committee, sixteen patients with diabetes mellitus and sixteen control subjects were included in this study. Three maxillary bone measurements (alveolar ridge height, bucco-palatal dimension, and trabecular bone density) were performed on selected CBCT cross-sectional images. These measurements were performed by two radiologists once and one of them carried out the measurements twice. Results:Thisstudy demonstrated that there was no significant difference between the normal and diabetic groups regarding all maxillary measurements except for the maxillary trabecular bone density of the normal group which was statistically lower than that of the diabetic group. Maxillary trabecular bone density was correlated significantly with most of the studied variablessuch as disease duration, type of DM medication, teeth extraction period, and gender.Conclusion:Diabetes is not an absolute contraindication for any maxillofacial surgeries and each diabetic patient indicated for any maxillofacial surgery should be evaluated independentlyregarding the maxillary bone quality and quantity as well as the contributing local factors.
\end{abstract}

Copy Right, IJAR, 2017,. All rights reserved.
Corresponding Author:-Sarah M. Kenawy.

Address:-Assistant Lecturer, Oral and Maxillofacial Radiology Department, Faculty of Dentistry, Cairo University, Cairo, Egypt. 


\section{Introduction:-}

Diabetes mellitus is the most common disorder of the endocrine system with an incidence of approximately 382 million worldwide. DM has been accompanied by the occurrence of a series of complications related to the skeletal system, such as the increase risk of fractures, poor osseous healing, and impaired bone regeneration potential(Aguiree et al., 2013; Rubin, 2015).

Extraction period, gender, genetic tendencies, systemic disorders, periodontal disease, and denture wearing may influence the degree of jaw bones resorption which may limit the surgical treatments potential. Additionally, the localization of the maxillary sinus may limit the potential of some treatments in the maxilla(Tutak et al., 2013).

In dental implantology, the assessment of bone quality and quantity, as well as localization of anatomical structures in the jaws, is crucial in treatment planning to avoid placement of implants into the worst bone, where failure is more probable(Nemtoi et al., 2013).

CBCT is an exciting development in dental and maxillofacial imaging. It has provided new images that continually add to the accuracy of diagnostic tasks of the maxillofacial region and has gained rapid acceptance in dentistry as it provided acceptable image quality associated with a lower exposure dose(Nemtoi et al., 2013; Rao et al., 2012).

Consequently, because of the increased number of Egyptian patients with diabetes mellitus and the influence of this systemic disease on the bone quality and quantity of the maxilla that has been investigated to a minor extent, this condition could not be neglected in the daily routine of dental practice. Therefore, the researchers performing this study were motivated to go through this work.

The aim of this study was to evaluate the maxillary jaw bone quality and quantity in a sample of Egyptian patients with DM compared to another nondiabetic one using CBCT and to assess the correlation between the maxillary jaw bone quality and quantity and other factors as glycated haemoglobin $(\mathrm{HbAlc})$ values, disease duration, type of DM medication, extraction period, and gender.

\section{Materials and Methods:-}

\section{Subjects:-}

Thirty-twopatientsattending the outpatient clinic of the Prosthodontic Department, Faculty of Oral and Dental Medicine, Cairo University, seeking prosthetic implant placement for their missing teeth and indicated for CBCT imaging, were selected and included in the study.Performance of this study was approved by the "Research Ethics Committee" of the Faculty of Oral and Dental Medicine, Cairo University.

Out of the 32 subjects who participated in this study, sixteen were diabetic patients "the study group" (with 43 maxillary edentulous bounded regions) and sixteen were healthy nondiabetics "the control group" (with 44 maxillary edentulous bounded regions).

During the clinical examination, patients had to meet the following inclusion criteria: age range from 30 to 65 years; presence of partially edentulous "bounded Class III and IV Kennedy's classification"; no current usage of drugs that could influence the bone density; absence of systemic diseases except DM type II for the diabetic group; no smoking or alcohol consumption; absence of bone lesions, fractures, deformity, or any previous surgeries within the edentulous area; and absence of pregnancy in female subjects.

Selected important data were collected from every diabetic patient as the disease duration, the type of DM medication, and the extraction period of every missing tooth.

\section{Laboratory Investigations:-}

All diabetic patients underwent blood sampling by venipuncture of the antecubital vein in order to determine HbA1c values on the same day of the radiographic examination.

\section{CBCT Imaging Acquisition:-}

All of the 32 participants were scanned for the regions of interest (ROI) indicated for implant placement using the "PLANMECA PROMAX ${ }^{\circledR}$ 3D MID \& PROFACE" CBCT scanner at the Oral and Maxillofacial Radiology 
Department, Faculty of Oral and Dental Medicine, Cairo University, under the following exposure parameters:90 $\mathrm{kV}, 10 \mathrm{~mA}$, exposure time of 18 seconds and $400 \mu \mathrm{m}$ voxel size.CBCT reconstructions were done by PlanmecaRomexis viewer 2.3.1 software (Planmeca, Helsinki, Finland).

\section{Measurements:-}

Three measurements were obtained for the region of each missing tooth in the bounded edentulous area by two oral radiologists with different experience in the field of oral and maxillofacial radiologywho performed the measurements once. In addition, one of the two radiologists performed the analysis twice.

Before performingthe measurements, a precise panoramic curve was accurately drawn on a selected axial cut at approximately the mid-root level of the maxillary teeth.The mesiodistaldimension for the region of each of the missing teethwas calculated by dividing the total mesiodistaldimension of the bounded edentulous area by the number of missing teeth on the reformatted panoramic image. On the same reformatted panoramic image, the width of each region was halved and a cross-sectional cut of $0.2 \mathrm{~mm}$ thickness going by this mid was selected to perform the previous mentioned measurements(Figs 1 - 6). This method for selecting the desired cross-sectional image was innovated by the researchers who performed this work.

\section{Alveolar Ridge Height:-}

On the selected cross-sectional cut, a line was plotted and measured perpendicular to another two lines drawn parallel and tangential to the alveolar crest and the nearest anatomical landmark (e.g., floor of the nasal cavity orthe maxillary sinus)(Zhang et al., 2015; Zheng et al., 2015)(Fig 7).

\section{Bucco-palatal Dimension:-}

On the same cross-sectional cut, at half the distance of the alveolar ridge height, a line was plotted and measured extending from the labial or buccal cortical plate to the palatal cortical plate. This line was drawn perpendicular to the line representing the alveolar ridge height and parallel to the other two lines drawn parallel and tangential to the alveolar crest and to the nearest anatomical landmark(Zhang et al., 2015)(Fig 8).

\section{Trabecular Bone Density:-}

On the same cross-sectional cut, the average trabecular bone density was measured within a circle (with a surface area of about $4.5 \mathrm{~mm}^{2}$ ); the center of this circle is the point of intersection between the line representing the alveolar ridge height and half of the line representing the bucco-palatal dimension (Fig 9).This method was innovated by the study researchers.

\section{Statistical Analysis:-}

A total sample size of 16 diabetic patients was sufficient to detect an effect size ( $\mathrm{f}=1.4$ ) which had been calculated based on study done by Nemoti et al.2013 with power $90 \%$ and 5\% significance level . In order to compare bone density with healthy control, similar number was recruited in the study. This sample size calculation was approved by the research committee in our faculty.

Intraclass correlation coefficients were calculated to determine the level of agreement between the dual records of the primary investigator and also the agreement between the two investigators' records. The level of agreement is specified according to Landis and Koch (1977) for interraterand intrarater reliability coefficients. Shapiro-Wilk test was used to determine thenormality of the data. Parametric unpaired Student's t-test andnonparametric MannWhitney $\mathrm{U}$ test were used to detect statistical differenceamong the groups for normal and non-normal distributed data, respectively. The correlations between the descriptive parameters of the diabetic group (gender, HbA1c\%, type of medication, extraction period, and disease duration) and the study measurements were evaluated by Spearman's correlationanalysis. Data were reported as means \pm standard deviation (SD). It was verified at $\mathrm{P} \leq 0.05$. The results are considered to be statistically significant if p-value was less than 0.05. All of the statistical analysis was performed with R statistical package, version 2.15.2 (26-10-2012).

\section{Results:-}

The level of intraobserverand interobserveragreements was almost perfect $(0.86$ to 0.97 and 0.9 to 0.94 respectively)regarding all measurements. 
The normality of the data was tested by Shapiro-Wilk test. It was found that the data for alveolar ridge height, bucco-palatal dimension, and trabecular bone density had normal distribution; therefore, parametric unpaired Student's t-test was used to detect statisticaldifference among the two groups. The data for disease duration, extraction period, and $\mathrm{HbA} 1 \mathrm{c} \%$ had non-normal distribution, and nonparametric Mann-Whitney U test wasused to detect the statistical difference among the diabetic group.

The mean values of the maxillary alveolar ridge height and the bucco-palatal dimension for the normal and diabetic groups were 13.67 and $13.84 \mathrm{~mm}$ and 9.57 and $8.64 \mathrm{~mm}$, respectively. There was no significant difference between the normal and diabetic groups regarding both measurements.

However, the mean trabecular bone density for the normal and diabetic groups was 71.16 and 221.9 , respectively, where the mean trabecular bone density of the normal group was statistically lower than that of the diabetic group (Fig 10).

For the correlation between the diabetic patients'gender and the obtainedmeasurements, it was found that that being a diabetic female was correlated with less trabecular bone density when compared to diabetic males $(\mathrm{rho}=-0.52, \mathrm{p}-$ value $=0.0006)$ (Fig 11). Meanwhile, the results showed no correlation between gender and alveolar ridge height (rho $=-0.05, \mathrm{p}=0.7782)$ and bucco-palatal dimension $(\mathrm{rho}=0.09, \mathrm{p}=0.5553)$.

Regarding the correlation between the diabetic patients' $\mathrm{HbA} 1 \mathrm{c} \%$ and the measurements, no correlation was found between $\mathrm{HbA} 1 \mathrm{c} \%$ and alveolar ridge height $(\mathrm{rho}=0.28, \mathrm{p}=0.07781)$, bucco-palatal dimension $(\mathrm{rho}=0.16, \mathrm{p}=$ $0.3088)$, and trabecular bone density ( $\mathrm{rho}=0.12, \mathrm{p}=0.4715)$.

Concerning the "disease duration" correlation analysis, the results showed that as the disease duration increases, the trabecular bone density increases significantly $(\mathrm{rho}=0.57, \mathrm{p}$-value $=0.0001)($ Fig 12). Meanwhile, the results show no correlation between disease duration and alveolar ridge height (rho $=0.19$, p-value $=0.2293$ ) and bucco-palatal dimension $(\mathrm{rho}=-0.001, \mathrm{p}$-value $=0.9943)$.

About the "extraction period" correlation analysis, the results showed that as the extraction period increases, the trabecular bone density decreases significantly $(\mathrm{rho}=-0.45$, p-value $=0.003)$ (Fig 13). Meanwhile, the results showed no correlation between extraction period and alveolar ridge height ( $\mathrm{rho}=-0.12$, $\mathrm{p}$-value $=0.4566)$ and bucco-palatal dimension $(\mathrm{rho}=-0.24, \mathrm{p}$-value $=0.1284)$.

Concerning the "type of DM medication" correlation analysis, the results showed that insulin therapy was correlated with less alveolar ridge height when compared to oral antiglycemic drugs $($ rho $=-0.35$, p-value=0.026) $($ Fig 14). It also showed that it was correlated with more trabecular bone density (rho=0.52, p-value=0.0006) (Fig 15). Besides, the results showed no correlation between the type of medication and bucco-palatal dimension $(\mathrm{rho}=0.06$, $\mathrm{p}-$ value $=0.6938)$.

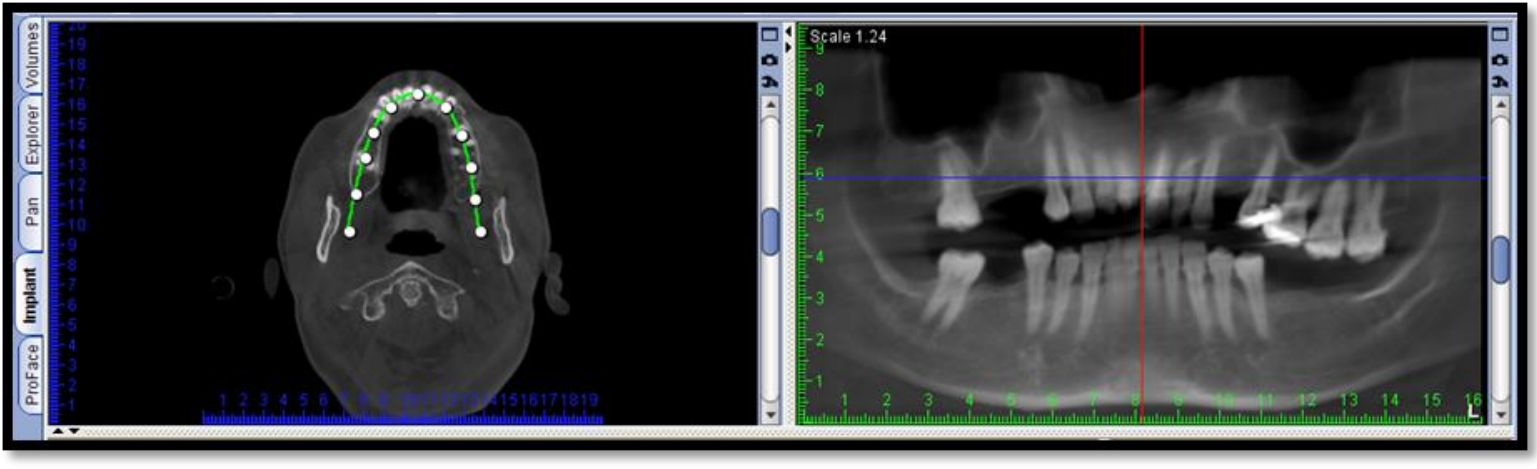

Figure 1: The selected CBCT axial image on which the panoramic curve of the maxilla was precisely drawn for this case. The CBCT reformatted panoramic image of the maxillary arch was created. 


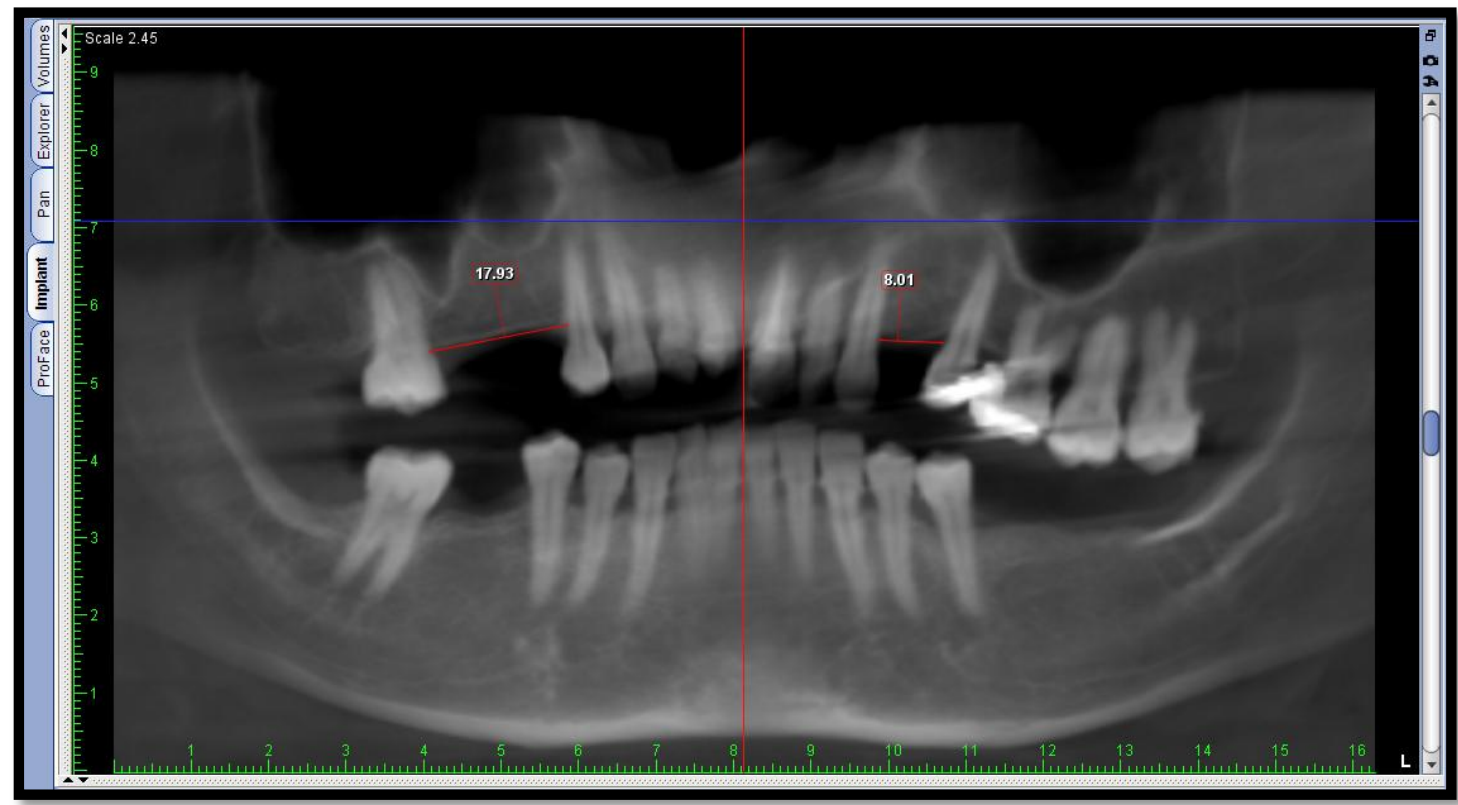

Figure 2:Measurement of the mesiodistal dimension of the maxillary bounded edentulous areas of this case on the CBCT reformatted panoramic image; it is equal to $8.01 \mathrm{~mm}$ for the left area (missing left maxillary $1^{\text {st }}$ premolar) and $17.93 \mathrm{~mm}$ for the right area (missing right maxillary 2nd premolar and first molar) in this case.

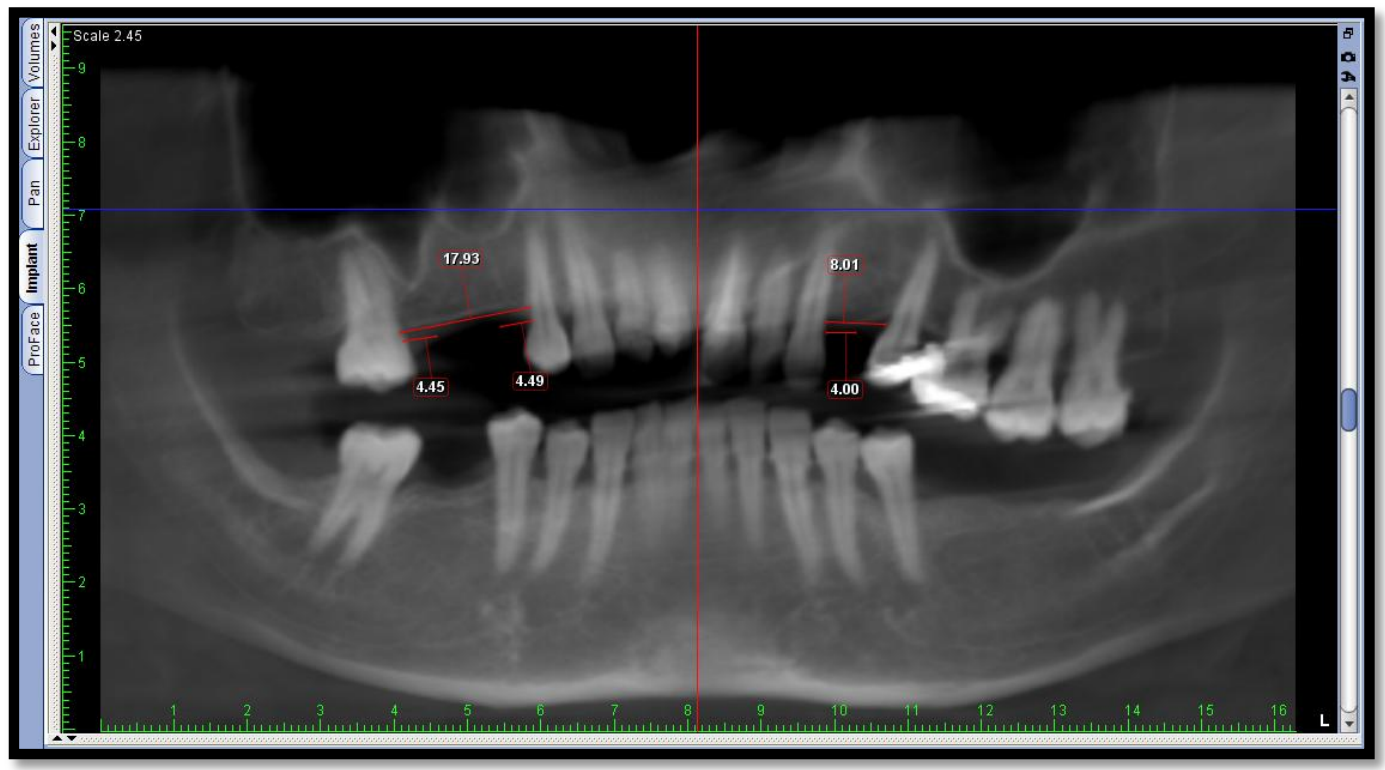

Figure 3: Measurement of half the mesiodistal dimension of each missing maxillary tooth region in the bounded edentulous areas of this case on the CBCT reformatted panoramic image; it is equal to $(8.1 / 2=4 \mathrm{~mm})$ for the left maxillary $1^{\text {st }}$ premolar region and equal to $(17.93 / 4=4.4 \mathrm{~mm})$ for the right $2^{\text {nd }}$ premolar and $1^{\text {st }}$ molar regions 


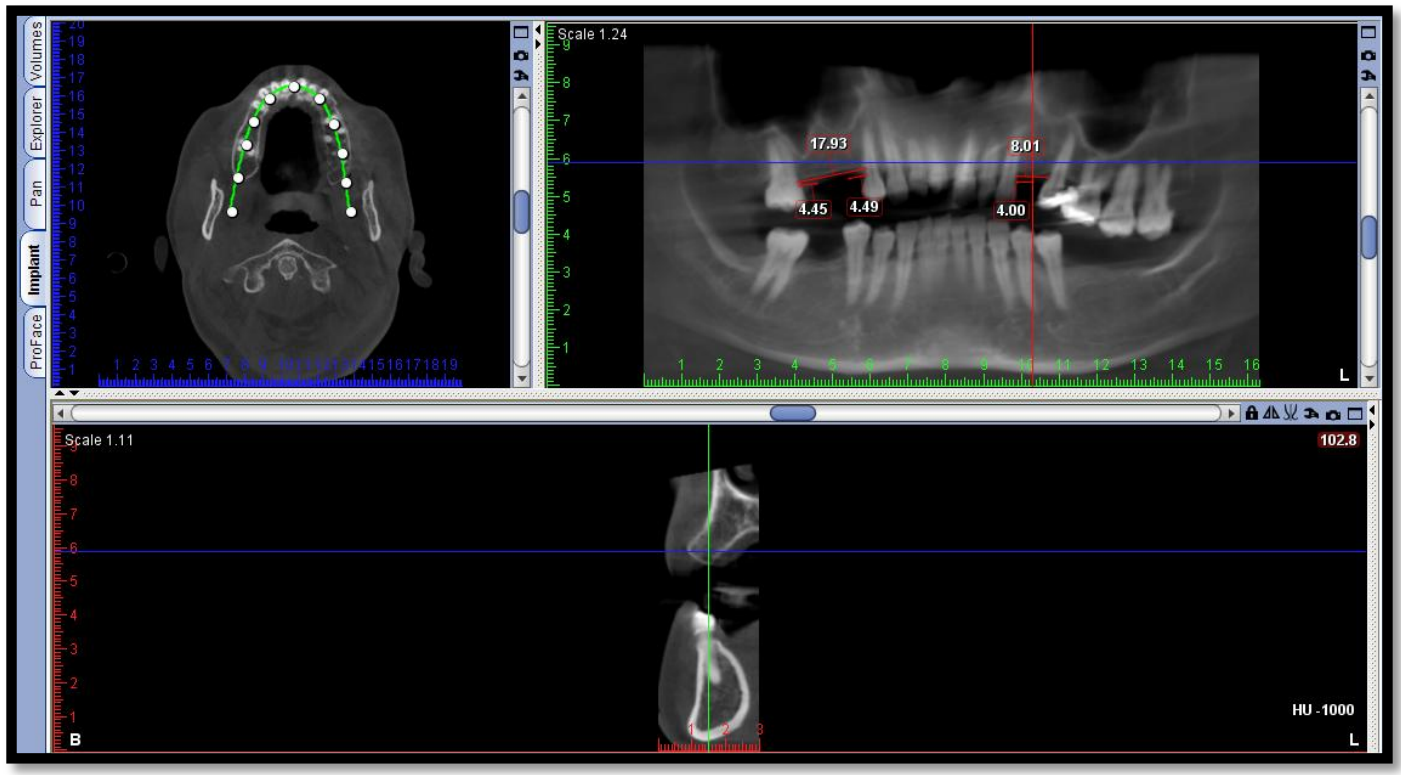

Figure 4: The selection of the desired CBCT cross-sectional image passing exactly by the mid of the left maxillary 1st premolar region in this case

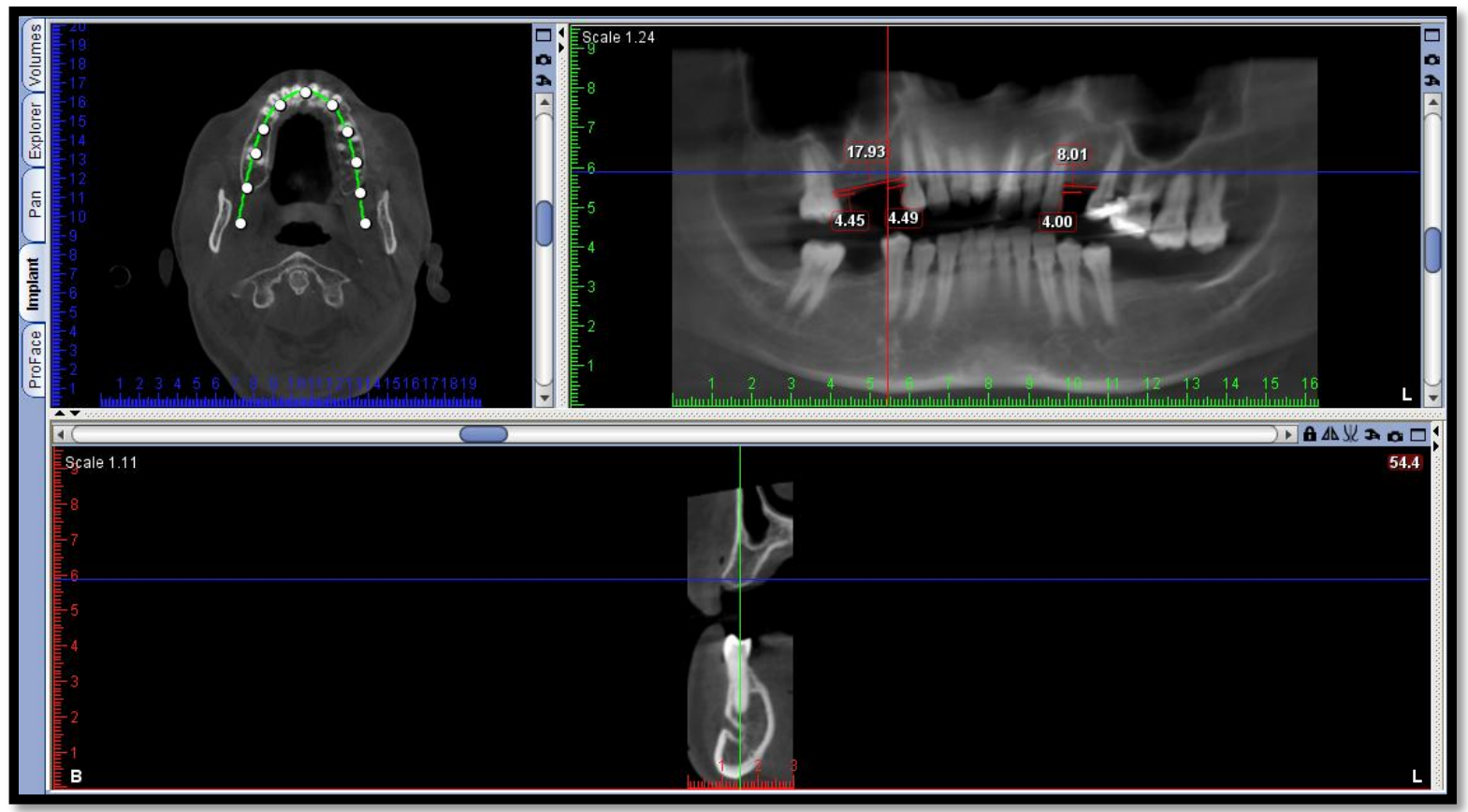

Figure 5: The selection of the desired CBCT cross-sectional image passing exactly by the mid of the right maxillary 2nd premolar region in this case. 


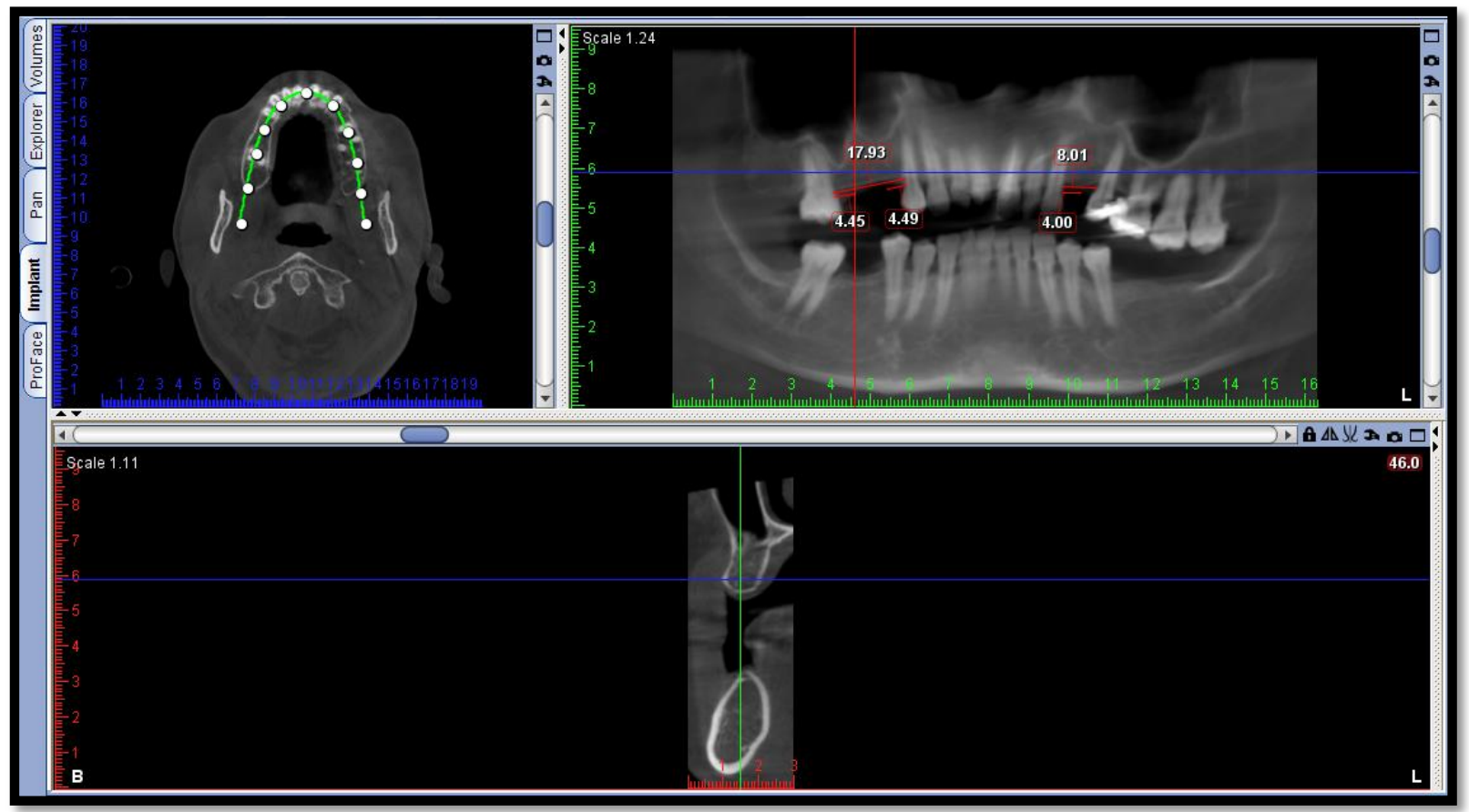

Figure 6: The selection of the desired $\mathrm{CBCT}$ cross-sectional image passing exactly by the mid of the right maxillary 1 st molar region in this case.

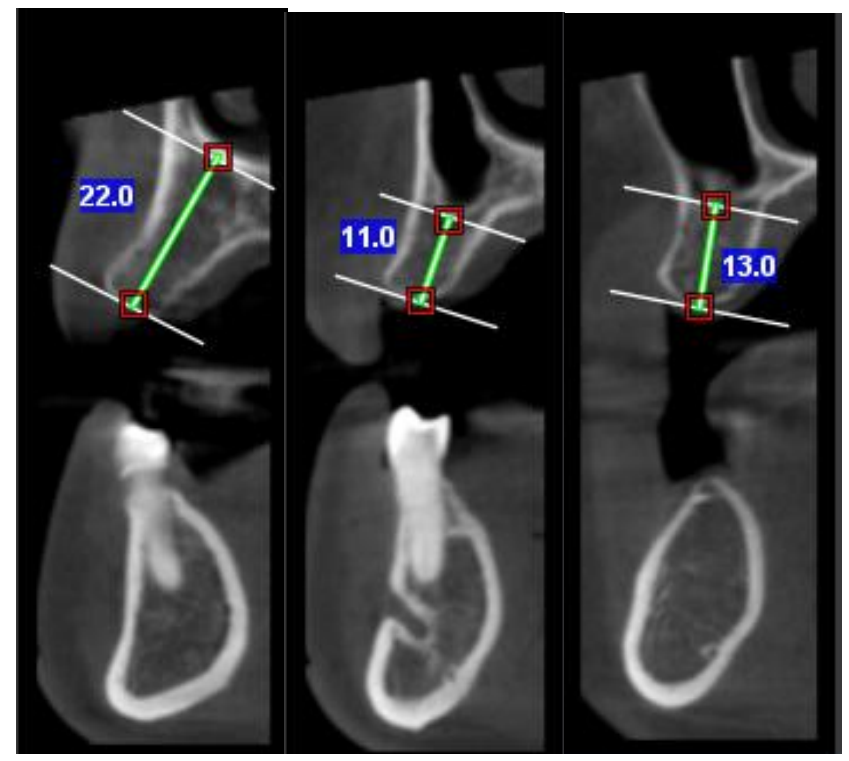

Figure 7: Measurement of the maxillary alveolar ridge height of this case on the selected CBCT coronal crosssectional image; it is equal to $22 \mathrm{~mm}$ for the left maxillary 1 st premolar region; $11 \mathrm{~mm}$ for the right maxillary $2 \mathrm{nd}$ premolar region and $13 \mathrm{~mm}$ for the right maxillary $1 \mathrm{st}$ molar region. 


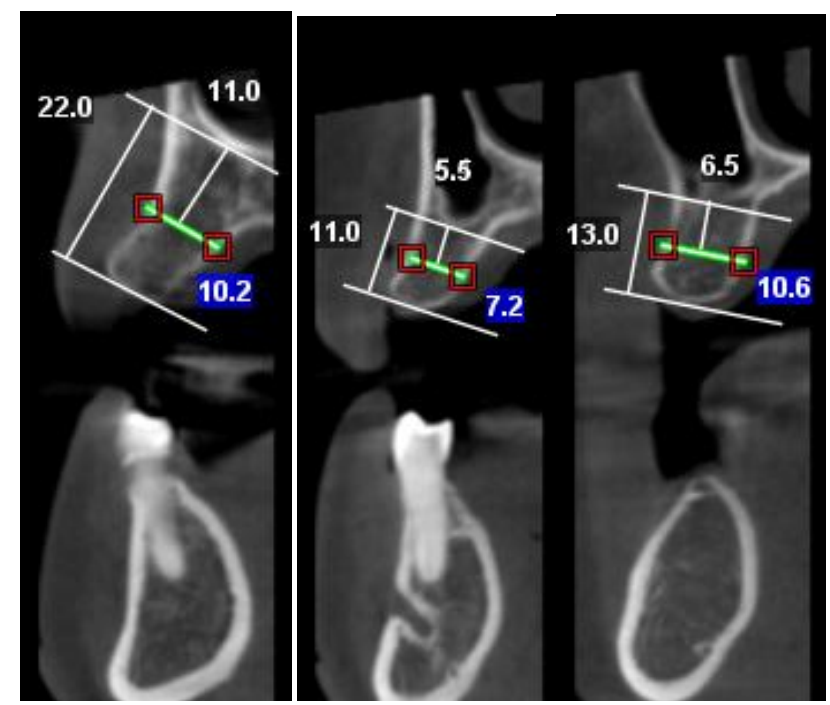

Figure 8: Measurement of the maxillary bucco-palatal dimension of this case on the selected CBCT coronal crosssectional image; it is equal to $10.2 \mathrm{~mm}$ for the left maxillary $1 \mathrm{st}$ premolar region; $7.2 \mathrm{~mm}$ for the right maxillary $2 \mathrm{nd}$ premolar region and $10.6 \mathrm{~mm}$ for the right maxillary $1 \mathrm{st}$ molar region.

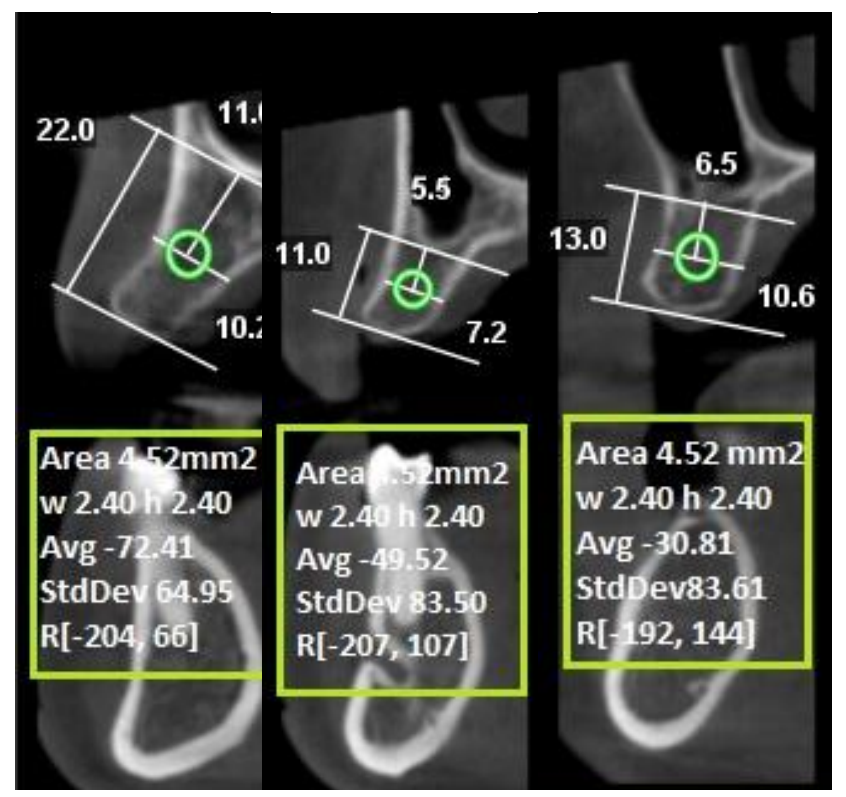

Figure 9: Measurement of the average maxillary trabecular bone density of this case on the selected CBCT coronal cross-sectional image; it is equal to -72.41 for the left maxillary 1 st premolar region; -49.52 for the right maxillary 2nd premolar region and -30.81 for the right maxillary 1 st molar region. 


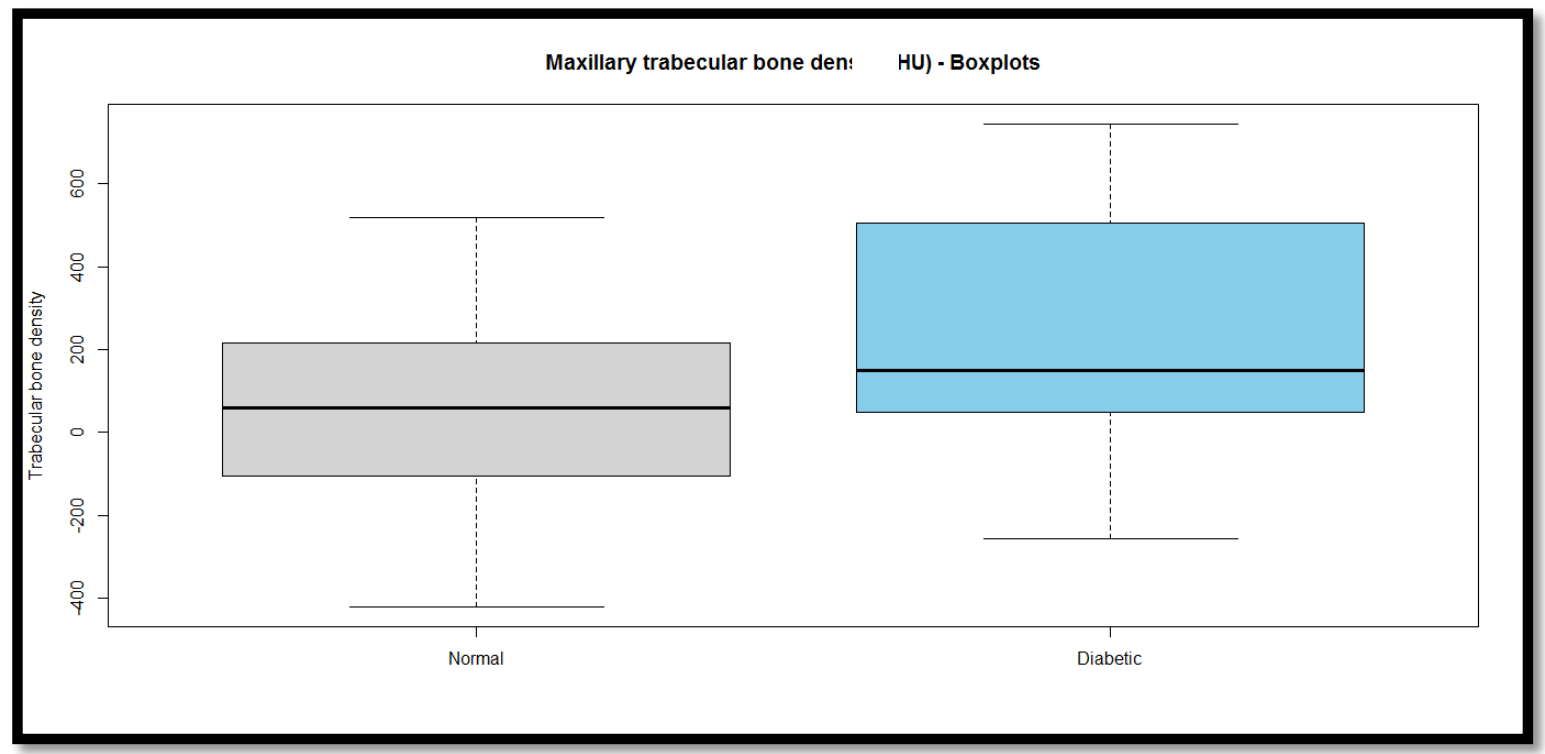

Figure 10: Maxillary trabecular bone density of the normal and diabetic groups - Boxplots.

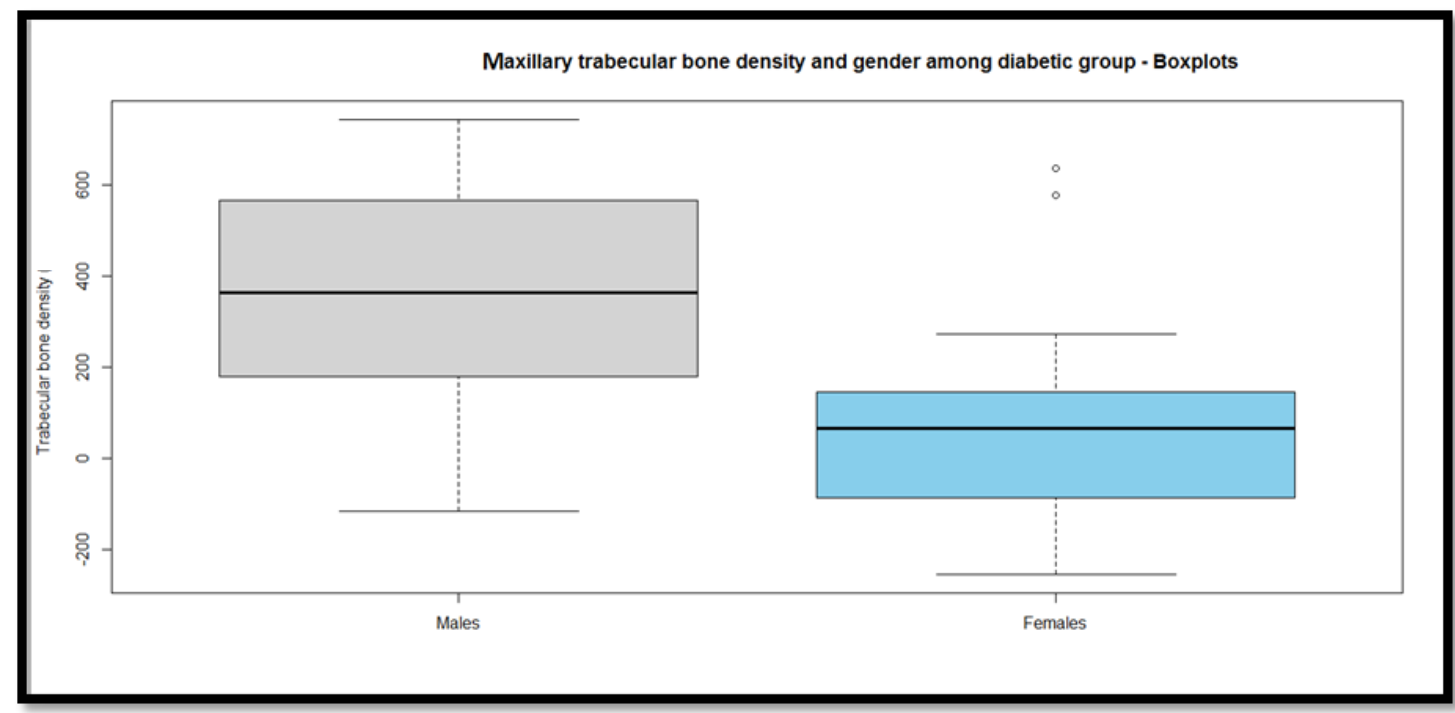

Figure 11: Maxillary trabecular bone density of the diabetic group according to gender - Boxplots. 


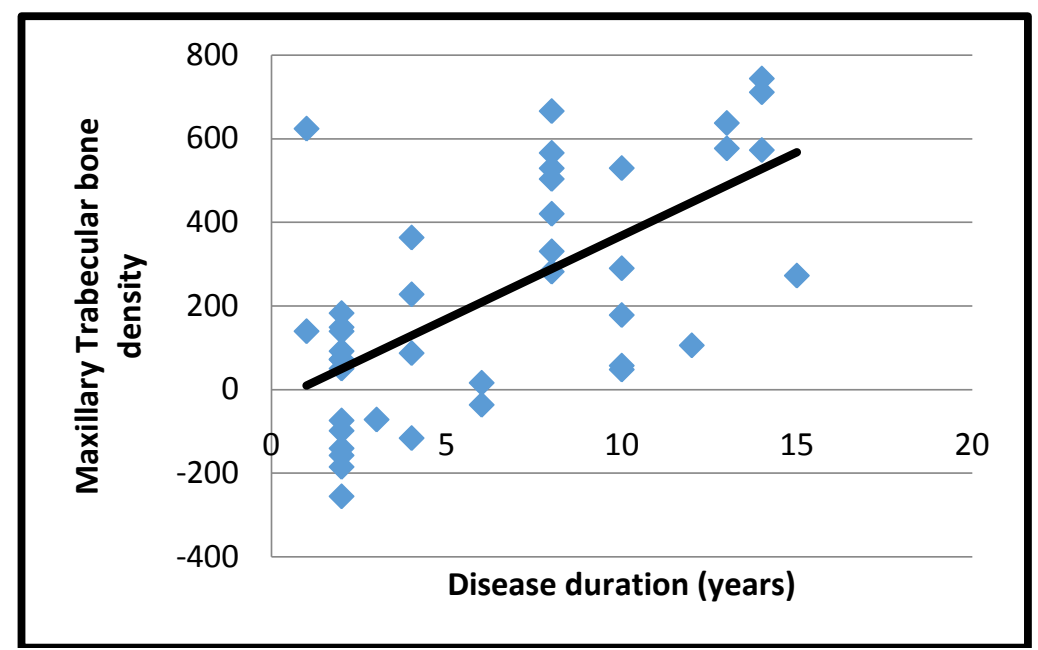

Figure 12: Relationship between disease duration and maxillary trabecular bone density among diabetic group.

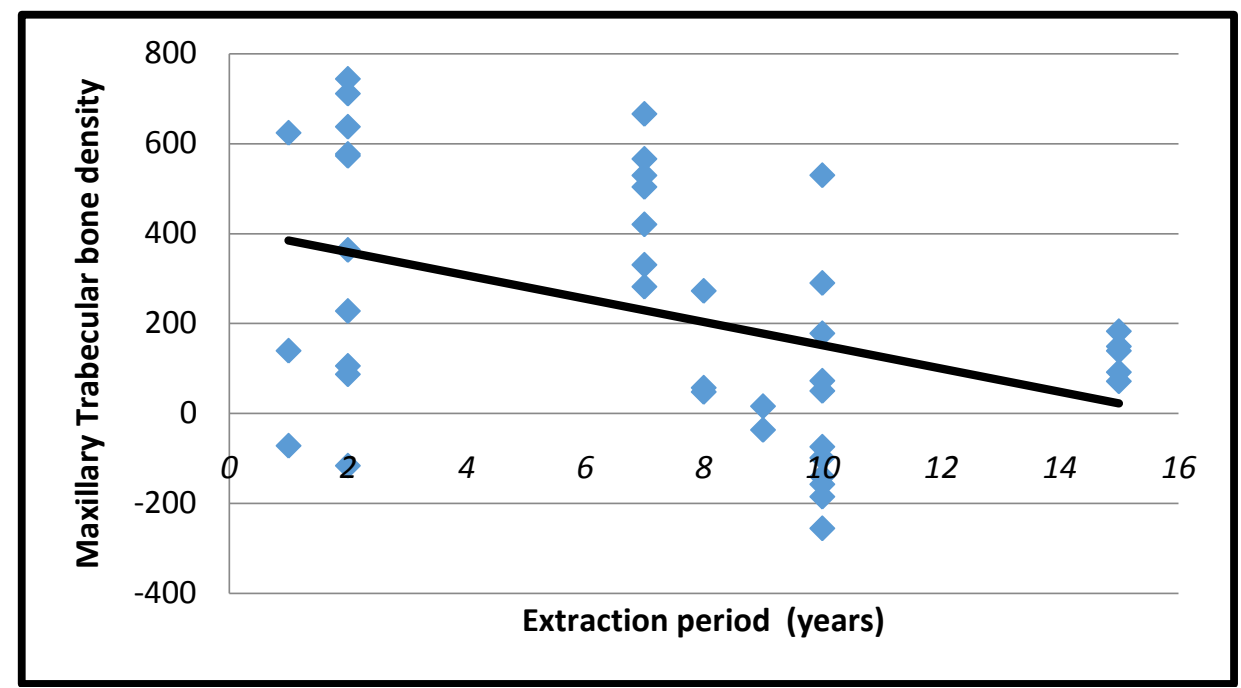

Figure 13: Relationship between extraction period and maxillary trabecular bone density among diabetic group. 


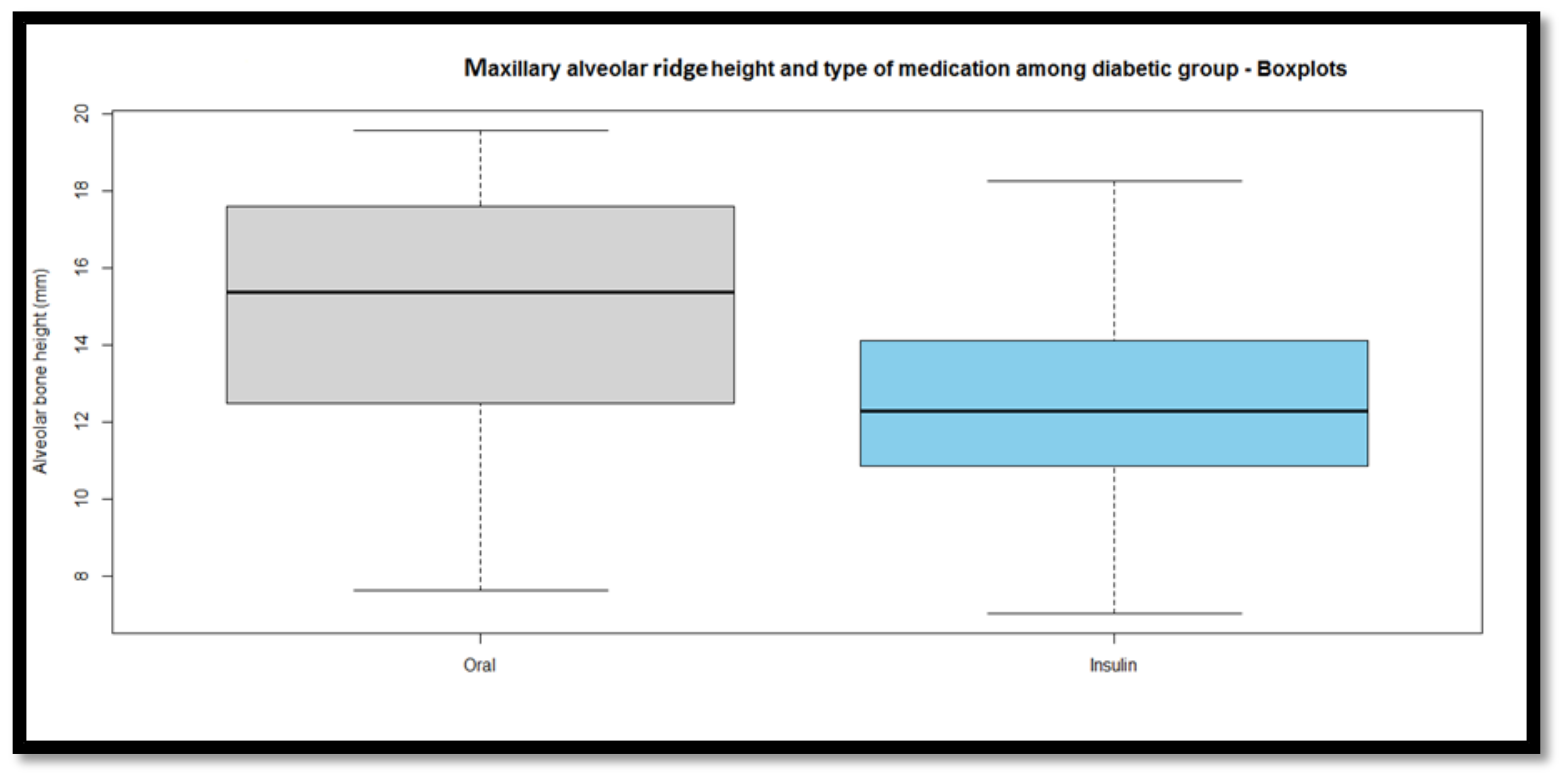

Figure 14: Maxillary alveolar ridge height of the diabetic group according to type of medication - Boxplots.

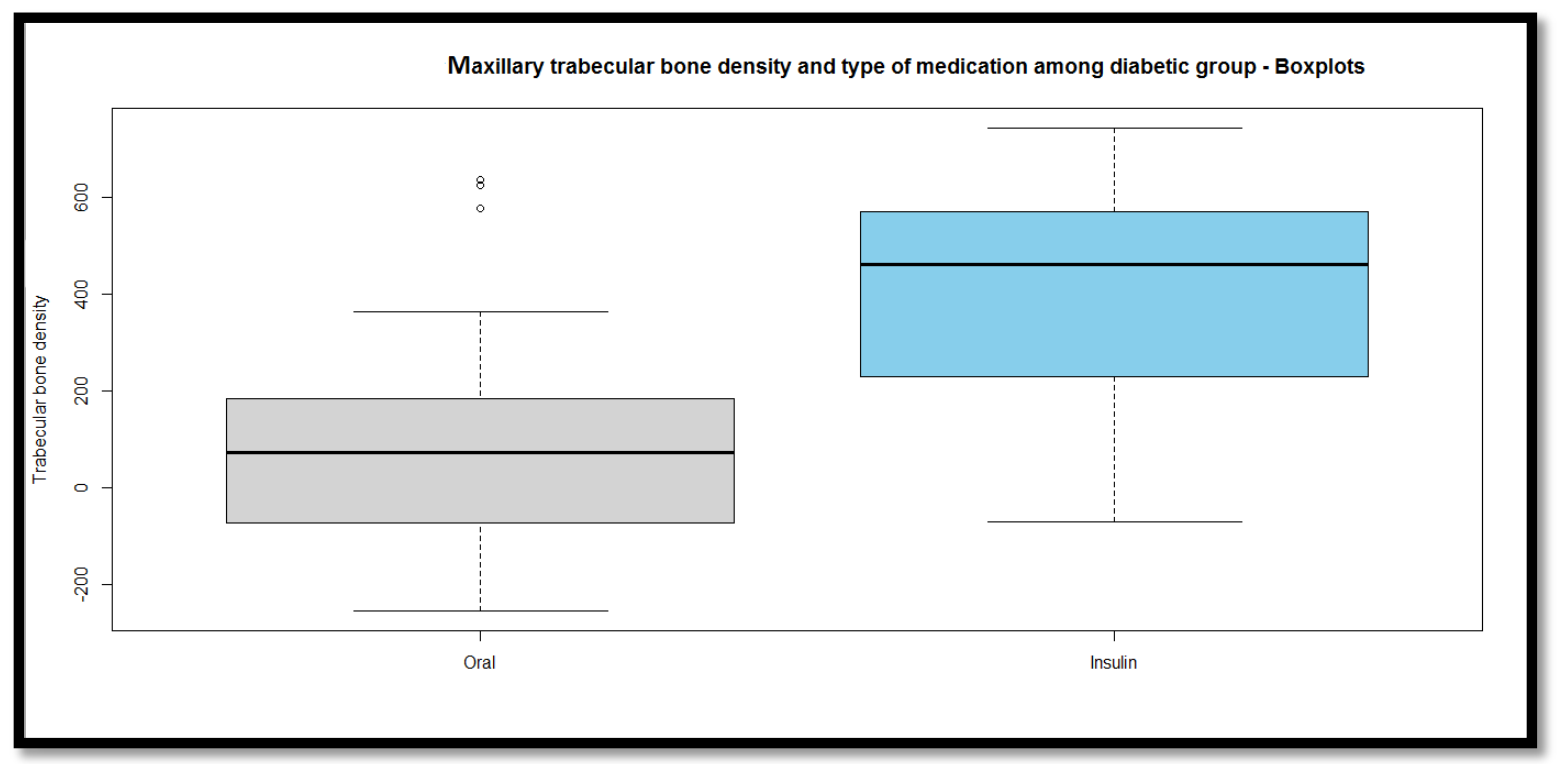

Figure 15: Maxillary trabecular bone density of the diabetic group according to type of medication - Boxplots.

\section{Discussion:-}

Preoperative assessment of both bone quality and bone quantity is necessary to optimize surgical procedures and also to increase thesuccess rate of dental implants. Moreover, the CBCT imaging and the interactive software enable bone quality and quantity measurements on CBCT slices(Gerlach et al., 2013).

Up to the authors' knowledge, no previous studies address the topic of evaluating the maxillary jaw bone quality and quantity in diabetic patients compared to another nondiabetic sample using CBCT and correlating between the maxillary jaw bone quality and quantity and other factors as glycated haemoglobin (HbAlc) values, disease duration, type of medication, extraction period, and gender.

Regarding the quantitative linear measurements, the results came along with a study(Hamozi \& Fattah, 2012) which showed that there was no statistically significant difference in the maxillary alveolar ridge height for both diabetic and nondiabetic groups in completely edentulous patients using digital panoramic radiographs. However, these 
results disagree with another study(Laith \&Kasiapan., 2009)that measured the maxillary alveolar ridge height using panoramic radiographs on completely edentulous denture wearing diabetic patients and found that diabetic patients have significantly less alveolar ridge height when compared with control subjects. This difference between the current study results and theirs can be explained on the basis that the increase in the alveolar ridge resorption is associated with an increase in the length of the edentulous area and with denture wearing(Al-Jabrah \& AlShumailan, 2013; Jagadeesh et al., 2013).No previous studies assessed radiographically the maxillary bucco-palatal dimension on diabetic patients to be compared with the studyresults.

Upon comparing the qualitative density measurements in this study, the mean trabecular bone density of the normal group was statistically lower than that of the diabetic group. Contradictingthe results of this work, Jolly et al., 2015 did not find any statistically significant difference in bone density at maxillary trabecular areas using axial CT images. However, the findings of the current workare in concordance with some studies(Kanis et al., 2005; Rubin, 2015)which had reviewed that the bone density values in T2DM patients were paradoxically greater than those in nondiabeticsubjects. This phenomenon is called "the paradox of bone fragility" which is explained by the fact that although DM type II is known to be accompanied with increase in fracture risk,bone density is only one of the determinants of bone mechanical strength. Other factors such as changes in bone thickness, trabecular microstructure, spatial distribution of the trabeculae, and intertrabecular space play an important role in determining the bone strength(Piscitelli et al., 2015).

Within the diabetic group, a correlation analysis between all measurements and HbA1c\%, disease duration, type of DM medication, extraction period, and gender was performed.

Concerning the correlation between HbA1c values and the quantitative linear measurements, the studyresults showed no correlation between them. Unfortunately, most of the studies that revealed these measurements were epidemiological and did not have access to HbA1c levels. The good control HbAlc values of most of the diabetic patients (ranging from 5.8 to $6.6 \%$ or 40 to $49 \mathrm{mmol} / \mathrm{mol}$ ) may explain the results where a good glycemic control in DM type II is accompaniedby decrease in bone resorption(Krakauer et al., 1995).

Regarding the correlation between HbA1c values and the qualitative density measurements, the results showed no significant correlations. These results come in agreement with Vestergaard, 2007, who published a meta-analysis searching the terms "diabetes", "fracture", and "bone density" and found that HbAlc levels as a measure of glycemic control do not seem to be associated with alteration in bone density.

The correlation between the disease duration and the quantitative linear measurements wasnonsignificant. Contradicting to the study results, Hamozi \& Fattah, 2012 found that the maxillary bone resorption of edentulous patients with T2DM progressed with increased period of the disease using digital panoramic radiography. The difference between the results of the two studies may be attributed to the fact that all patients included in their sample were denturewearers, which might have played a role in accelerating the alveolar bone loss.

Regarding the correlation between the disease duration and the qualitative density measurements, the results were significantly correlated. This significance can be explained on the basis that the bone density values in T2DM patients are paradoxically greater than nondiabetic controls and hence the increase in disease duration is associated with a rise in bone density(Kanis et al., 2005; Piscitelli et al., 2015; Rubin, 2015; Wakasugi et al., 1993).

On correlating the type of DM medication and the quantitative linear measurements, the results showed that insulin therapy was correlated significantly with less alveolar ridge height when compared to oral antiglycemic drugs. This can be attributed to an accidental fact in the investigated sample as most of the diabetic patients on insulin therapy included in the study were older than those on oral antiglycemics.

On correlating the type of DM medication with qualitative density measurements, insulin therapy showed direct significant correlation. This comes in accordance with a study(de Morais et al., 2009) which concluded that insulin therapy may have an anabolic effect on bone using digital subtraction radiographs. Additionally, Piscitelli et al., 2015 who reviewed that direct negative effects on bone have been reported for some oral antiglycemic drugs and current insulin usage was not associated with bone loss. Moreover, some oral antiglycemic agents' skeletal effects are mediated by peroxisome proliferator-activated receptor gamma activation that depends on the cell fate 
determination of mesenchymal stem cells toward adipogenesis rather than osteogenesis, thus leading to a reduction of osteoblastic bone formation(Kawai, 2013).

Regarding the teeth extraction period correlation with all the quantitative linear measurements, the results showed no significant correlations. This can be justified by the fact that the teeth extraction period of most of the patients exceeded 2 years. A published review(Van Der Weijden et al., 2009) stated that the size (height and width) of the residual ridge is decreased most rapidly in the first six months and then the residual ridge bone resorption activity continues throughout life at a dramatically slower rate. Additionally, all the patients were partially edentulous where the resorptive changes after tooth extraction on the alveolar ridge depend on the number and position of the remaining teeth in the jaw where the alveolar ridge resorption increases in totally edentulous patients than in partially edentulous ones(Knezoviê-zlatariê, 2002). However, the results showed that as the extraction period increases, the trabecular bone density decreases significantly.

For the correlation between gender and all the quantitative linear measurements, the results showed no correlations. Conversely, these results disagree with a study(Zhang et al., 2015)which found that males demonstrated significantly larger alveolar width compared to females for anterior maxilla.

Concerning the correlation between gender and the qualitative density measurements, the results showed that being a diabetic female is correlated significantly with less trabecular bone density when compared to diabetic males. These results can be explained on the basis that most of the diabetic patients in this study were over 45 years of age where osteoporosis is more common in women than in men and accelerated bone loss occurs in women after menopause because estrogen withdrawal increases the rate of bone remodeling. Osteoclastogenesis is amplified by estrogen deficiency via cytokines and growth factors. Thus, increased bone remodeling occurring after menopause causes less bone formation and more significant negative bone balance, leading to trabecular thinning that characterizes postmenopausal osteoporosis(Liu, 2013).

\section{Conclusion:-}

Diabetes is not an absolute contraindication for implant placement or any maxillofacial surgeries. Therefore, within the limitation of the current study, each diabetic patient indicated for any maxillofacial surgery should be evaluated carefully and independently regarding the bone quality and quantity as well as the contributing local factors such as $\mathrm{HbA} 1 \mathrm{c}$ values and the disease duration.

Maxillary trabecular bone density in diabetic subjects correlated significantly with most of the studied variables as disease duration, type of DM medication, teeth extraction period, and gender and was more sensitive and susceptible to variability. So, an accurate and detailed assessment of the maxillary jaw bone quality should be performed prior to any surgical intervention.

\section{Acknowledgment:-}

The authors acknowledge the Prosthodontic Department, Faculty of Oral and Dental Medicine, Cairo University, for their constant help through this work.

\section{Duality of Interest:-}

No potential conflicts of interest relevant to this study were reported.

\section{Funding:-}

This research did not receive any specific grant from funding agencies in the public, commercial, or not-for-profit sectors.

\section{References:-}

1. Aguiree F, Brown A, Cho NH, Dahlquist G, Dodd S, Dunning T, Hirst M, Hwang C, Magliano D, Patterson C, Scott C, Shaw J, Soltesz G, Usher-Smith J, W. D. (2013). IDF Diabetes Atlas: sixth edition. Basel, Switzerland: International Diabetes Federation. http://doi.org/2-930229-80-2

2. Al-Jabrah, O. A., \& Al-Shumailan, Y. R. (2013). Association of complete denture wearing with the rate of reduction of mandibular residual ridge using digital panoramic radiography. International Journal of Dental Research, 2(1), 20-25. 
3. de Morais, J. A. N. D., Trindade-Suedam, I. K., Pepato, M. T., Marcantonio Jr, E., Wenzel, A., \& Scaf, G. (2009). Effect of diabetes mellitus and insulin therapy on bone density around osseointegrated dental implants: a digital subtraction radiography study in rats. Clinical Oral Implants Research, 20(8), 796-801.

4. Gerlach, N. L., Meijer, G. J., Borstlap, W. A., Bronkhorst, E. M., Bergé, S. J., \& Maal, T. J. J. (2013). Accuracy of bone surface size and cortical layer thickness measurements using cone beam computerized tomography. Clinical Oral Implants Research, 24(7), 793-7.

5. Hamozi, S. M., \& Fattah, A. A. (2012). Digital panoramic assessment of maxillary implant insertion areas among controlled type 2 diabetic patients. J Bagh College Dentistry, 24(1), 61-63.

6. Jagadeesh, M. S., Patil, R. a, \& Kattimani, P. T. (2013). Clinical Evaluation of Mandibular Ridge Height In Relation To Aging and Length of Edentulism. Journal of Dental and Medical Sciences, 3(4), 44-47.

7. Jolly, S. J., Hegde, C., \& Shetty, S. (2015). Assessment of maxillary and mandibular bone density in controlled type II diabetes- A computed tomography study. J Oral Implantol . 2015 Jul 8;41(4):400-5.

8. Kanis, J. A., Borgstrom, F., De Laet, C., Johansson, H., Johnell, O., Jonsson, B., ... Khaltaev, N. (2005). Assessment of fracture risk. Osteoporosis International: A Journal Established as Result of Cooperation between the European Foundation for Osteoporosis and the National Osteoporosis Foundation of the USA, $16(6), 581-9$.

9. Kawai M. (2013). Adipose tissue and bone: role of PPAR $\gamma$ in adipogenesis and osteogenesis. - PubMed - NCBI. Horm Mol Biol Clin Investig, 15(3), 105-113.

10. Knezoviê-zlatariê, D. (2002). Resorptive Changes of Maxillary and Mandibular Bone Structures in Removable Denture Wearers. Bone, 36(6), 261-265.

11. Krakauer, J. C., Mckenna, M. J., Buderer, N. F., Rao, D. S., Whitehouse, F. W., \& Parfitt, A. M. (1995). Bone Loss and Bone Turnover in Diabetes. Diabetes, 44(August 1994), 775-82.

12. Laith, M., Kasiapan., S. (2009). Residual Alveolar Ridge Resorption in Completely Edentulous Patients Influenced By Pathophysiologic Factors. Dentika Dental Journal, 14(1), 29-36.

13. Liu, J. (2013). Gender differences in mandibular bone mineral distribution with aging. doi:10.1017/CBO9781107415324.004.

14. Nemtoi, A., Ladunca, O., \& Dragan, E. (2013). Quantitative and qualitative bone assessment of the posterior mandible in patients with diabetes mellitus: a cone beam computed tomography study. ... Medico-Chirurgicala a ..., 117(4), 1002-1008.

15. Piscitelli, P., Neglia, C., Vigilanza, A., \& Colao, A. (2015). Diabetes and bone: biological and environmental factors. Current Opinion in Endocrinology, Diabetes and Obesity, 22(6), 439-445.

16. Rao, G. V., Rao, S. A., Mahalakshmi, P. M., \& Soujanya, E. (2012). CONE BEAM COMPUTED TOMOGRAPHY - AN INSIGHT BEYOND EYESIGHT IN CLINICAL DENTISTRY. Innovative Journal of Medical and Health Science, 2, 74-80.

17. Rubin, M. R. (2015). Bone cells and bone turnover in diabetes mellitus. Current Osteoporosis Reports, 13(3), $186-91$.

18. Tutak, M., Smektała, T., Schneider, K., Gołębiewska, E., \& Sporniak-Tutak, K. (2013). Short dental implants in reduced alveolar bone height: a review of the literature. Medical Science Monitor: International Medical Journal of Experimental and Clinical Research, 19, 1037-42.

19. Van Der Weijden, F., Dell'Acqua, F., \& Slot, D. E. (2009). Alveolar bone dimensional changes of postextraction sockets in humans: A systematic review. Journal of Clinical Periodontology, 36(12), 1048-1058.

20. Vestergaard, P. (2007). Discrepancies in bone mineral density and fracture risk in patients with type 1 and type 2 diabetes--a meta-analysis. Osteoporosis International: A Journal Established as Result of Cooperation between the European Foundation for Osteoporosis and the National Osteoporosis Foundation of the USA, 18(4), 427-44.

21. Wakasugi, M., Wakao, R., Tawata, M., Gan, N., Koizumi, K., \& Onaya, T. (1993). Bone mineral density measured by dual energy x-ray absorptiometry in patients with non-insulin-dependent diabetes mellitus. Bone, 14(1), 29-33.

22. Zhang, W., Skrypczak, A., \& Weltman, R. (2015). Anterior maxilla alveolar ridge dimension and morphology measurement by cone beam computerized tomography (CBCT) for immediate implant treatment planning. BMC Oral Health, 15(1), 65.

23. Zheng, X., Teng, M., Zhou, F., Ye, J., Li, G., \& Mo, A. (2015). Influence of Maxillary Sinus Width on Transcrestal Sinus Augmentation Outcomes: Radiographic Evaluation Based on Cone Beam CT. Clinical Implant Dentistry and Related Research, 1-9. 